\title{
Factors Affecting Taxpayers' Decisions in Saving Tax by Investing in Tax Saving Bonds: A Study in U.P. State, India
}

\author{
Manoj Kumar ${ }^{1}$, Vikas Anand ${ }^{2}$ \\ ${ }^{1}$ Victoria University College, \\ Department of Business Studies, \\ 12(A), U Tun Myat Rd, Tarmwe Tsp. \\ Yangon, Myanmar \\ E-mail: mkumar75@gmail.com \\ ${ }^{2}$ Global Institute of Management, \\ Department of Financial Studies, \\ Amritsar, Punjab, India \\ E-mail: vikasanand_2001@yahoo.com
}

\begin{abstract}
While enabling oneself eligible for deduction from the partial or total tax liabilities, choosing the best investment is one of the most important decisions a taxpayer in India can make. One of the alternative investment avenues, tax saving bonds in India is considered as an option to serve any investor with two purposes: tax saving and investment growth with almost zero risk. Majority of the investors in India consider investing in a financial instrument as a very critical decision when it comes to the risks in their investments. The present study is to explore how different attributes of a financial instrument (such as tax saving bonds) are perceived by the investors of a province in India. Results in this study indicate that while taxpayers continue to place too much emphasis on return from the investment and in particular tax benefits, interact with other critical firm and product specific attributes such as past performance, lock-in period, etc., to influence perceptions and evaluations of tax saving bonds.
\end{abstract}

Keywords: investors, tax saving bonds, tax free bonds, India

JEL codes: G10, G11, G21, N25

\section{Introduction}

Tax saving bonds (TSBs) in India is proposed as an investment instrument to tax payers to lessen their tax liabilities either partially or completely (subject to tax slabs and income of the individuals in a particular financial year). These bonds are generally issued either by the government (such as Reserve Bank of India bonds on behalf of the Government of India) or by certain public banks (such as the State Bank of India) and private banks (such as ICICI, IDBI, HDFC). Unlike other tax savings instruments, tax saving bonds, unfortunately, are not very popular. The reasons might be higher inflation rates in the country, longer mandatory lock-in period (the minimum period for which investments are to be kept invested in), lower effective return on investment, etc. 
In the Indian Income Tax Act of 1961, a new section 80CCF was proposed in the union budget of 2010. This section (80CCF) allows an individual taxpayer to invest in Long Term Infrastructure Bond and get tax exemption of INR (short for Indian Rupees, $\$ 1$ = INR 60.00 approximately) 20,000/- per financial year. This limit is above the current income tax section 80C limit (of INR 100,000/-) for taxexempt benefits by investing into notified instruments.

The main purpose of proposing this new section (80CCF) in the budget was to promote infrastructure development in the country. Looking at the growing demand and need for better infrastructures in the country, the government proposed that the savings made by subscribing these bonds are to be utilized for infrastructure financing purposes.

This section (80CCF) serves mainly two purposes to investors: a) tax exemption from the actual liabilities, and b) guaranteed return from the investment at a fixed interest rate and for the life of the bond. By investing in these infrastructure bonds, the investors get assured rate of returns and, usually, yield of these bonds varies from 7.00 to $9.50 \%$ per annum. Generally, the maturity period of the bonds varies from 10 to 15 years and the minimum lock-in period is for 5 years. After the lock-in period, the individual investor has the option to exit from these infrastructure bonds through either a secondary market trading or a buy back option as specified by the bond issuer. Though, offering comparatively high interest rates, the biggest drawback in the case of infrastructure bonds is that the interests earned are taxable in the hands of investors and the hurdle of the lockin period, etc.

The reserve Bank of India allows Foreign Institutional Investors (FIIs) to invest up to USD 25 billion in infrastructure bonds and debentures of Indian infrastructure companies. Since FIIs are enthusiastically participating in infrastructure developments in India, how domestic investors and taxpayers see it from the investment perspective as well as being part of the development is a question for which an answer has to be found.

The main objective of this research is to explore how various attributes of TSBs impact Taxpayers' (investors') fund evaluations (attitude) and investment intentions.

\section{Review of Literatures}

Dick and Basu (1994); Gerpott et al. (2001); and Lee and Cunningham (2001) explored the relationship between satisfied customers and firms providing different financial products. Their studies revealed that the satisfied customers opted to maintain their relationship with the firms in which these customers invested and willed to continue in investing. Gordon (1991) in his study explores the $\mathrm{EE}$ and $\mathrm{HH}$ series US savings bonds that were present at that time. $\mathrm{He}$ concluded that if an investor considers the safety factor and/or the tax deferral 
feature and/or the competitive variable interest rate, then these bonds are an investment worthy of consideration.

Singh \& Chander (2004) studied the perceptions of investors towards mutual funds and results of their study revealed that in majority five factors were critical to investors to make an investment decision. These five critical factors were:

1. Better Investment Opportunities

2. Risk, Return and Performance of Mutual Fund (and Firm)

3. Professional Expertise of Managers of Mutual Funds

4. Benefits and Transparency in Disclosing Information

5. Listing Status (the investors expected open-ended mutual funds should also be listed on stock exchanges like close-ended mutual funds)

Athma and Kumar (2007) in their research on a sample of 200 respondents tested if there was any association between Residency and Attributes of the product. They also studied to identify the factors affecting consumers' decision in selecting Life Insurance Products. Clark et al (2012) surveyed over 2300 participants, who were members in the defined contribution pension plan of a London based international bank. The study predicted the significance of retirement saving of the relation between the respondents' socioeconomic status and risk preference. Vardhini (2012) compares both types of investment instruments, i.e. tax saving bonds (TSBs) and tax-free bonds (TFBs), with other debt instruments in India and concludes that post-tax yields are much superior in TSBs and TFBs as compared to most other debt instruments. A tax saving bond is one in which the initial investment is exempted from tax liabilities but interest earned, during the period of holding that instrument, is not exempted from tax. Dash \& Sood (2013) explored various demographic characteristics and customers' perceptions towards investing into various life insurance products in India. They concluded that the life insurance company (Life Insurance Corporation, the biggest insurer in India and the subject company in the study) has to redevelop strategies to convince the customers to buy the insurance products as a saving instrument or pension package.

Parasuraman, A., et al. (1985) in their exploratory research revealed several insights and suggestions with regards to the consumers' perceptions about service quality. Researchers concluded that there are 10 determinants that consumers use to form perceptions about the quality. We followed their research in developing basic framework (objectives) of our study.

In fact, there is a plethora of studies, research papers and literatures available on investors' attitude towards investments in Mutual Funds, Equity Linked Saving Schemes (ELSS), Insurance and many other financial instruments but not a single study, in the researchers' knowledge, was conducted on Tax Saving Bonds (TSBs) so far. Therefore, this is an opportunity to the researchers to explore empirically 
in taxpayers' attitude towards TSBs as an investment option and what factors are affecting their decisions for such investments. Also, this is an opportunity for the readers from the other geographic locations to learn about this investment instrument in India.

\section{Objectives}

The purpose of the study is to explore (1) the taxpayers' perception towards Tax Saving Bonds (TSBs); and (2) what factors are influencing their fund evaluations and investment intentions. Therefore, the following are the objectives formulated for this study:

1. To explore and understand desirable attributes of TSBs.

2. To analyze investors' perception towards TSBs as an investment option.

3. To explore the critical factors associated with TSBs which may have an impact on investors' investment decisions.

\section{Methodology}

The present study is primarily based upon primary data collected by conducting a survey using a structured questionnaire. The design of study has three sub sections:

1. Instrument (Questionnaire) Development

2. Sample Selection, Pilot Study, Redesigning Questionnaire, Sampling Procedures and Data Collection

3. Application of Statistical Tools (in the light of objectives of the study) for outcomes and results.

\subsection{Instrument Development}

A structured questionnaire with two sections is developed as an instrument for this study. The first section has questions to collect demographic data of the respondents and the second section has questions to collect responses on the different attributes of TSBs. The second section of the questionnaire comprised of 16 questions related to 16 critical attributes of TSBs for the study. All the attributes are measured by responses on a 5-point rating Likert scale, where rating 1 would mean Totally Disagree and rating 5 would mean Totally Agree. Annexure 1 shows a sample of questions from section two of the questionnaire. The surveys were conducted between June 2012 and November 2012.

\subsection{Sample Selection, Sample Procedures and Data Collection}

Initially, a pilot study was conducted in the Aligarh district (west region) of Uttar Pradesh state in India and data was collected to improve the questionnaire. In the second phase, using the convenience sampling method, 350 respondents from the Aligarh district (225 males and 125 females) were surveyed to fill in the questionnaire. After careful screening of data validation, out of the total response received (267 respondents), only 254 questionnaires were found to be complete 
and suitable for the analysis. The outcomes and results of this study are based upon 250 questionnaires that were randomly selected $(71 \%$ of original responses).

Furthermore, the guidelines below were also followed in the screening process:

a. Exclude the students, considering that they are either unemployed or do not fall under tax payer category of Income Tax Act, 1961 of India

b. Exclude respondents with salary equal to or below INR 200,000 as there is $100 \%$ tax exemption for incomes up to INR 200,000 as per existing income tax laws (financial year ending 31st March, 2013) in India.

\subsection{Analytical Tools}

The collected data was classified, sorted, tabulated, and analyzed using statistical tools like mean, standard deviation and correlation analysis, etc. to make the study meaningful. To measure internal consistency of the data, Cronbach's Alpha test for reliability was applied. Further, to determine whether objectives of the study are met or not, Kaiser-Meyer-Olkin (KMO) test for measure of sample adequacy, Bartlett's test of sphericity, and Factor Analysis for dimension reduction were employed. All the results in this study are the outcomes of the above tests using SPSS V.17 software (George and Mallery, 2012).

\section{Limitation of the Study}

The study is based upon the primary data collected through a survey conducted on 350 tax paying respondents, who were also opting for other scheduled investment opportunities for tax saving benefits under different sections of the Indian Income Tax Act (1961). The results and implications of this study are subjected to the limitations of sample size, a selected class of taxpayers, the geographic location of respondents (only the Aligarh District in the western region of Uttar Pradesh State of India), psychological and emotional characteristics of surveyed population and certain imposed conditions etc.

This study can be extended onto a wider range of population in the country to generate further useful results, which might be used by government or banks in India for better offers/solutions while encouraging or creating more awareness among investors.

\section{Analysis of Data}

The table below represents the attributes (variables) used in the study:

\begin{tabular}{lc}
\hline \multicolumn{1}{c}{ Attribute } & Notation \\
\hline \hline Risk in Investment & $\mathrm{F} 1$ \\
\hline Return from Investment & $\mathrm{F} 2$ \\
\hline Cost of buying Asset (Entry/Exit Load, Tax) & $\mathrm{F} 3$ \\
\hline Tax Benefits from Investment & $\mathrm{F} 4$ \\
\hline Fringe Benefits & $\mathrm{F} 5$ \\
\hline
\end{tabular}




\begin{tabular}{lc}
\hline \multicolumn{1}{c}{ Attribute } & Notation \\
\hline \hline Reputation of seller Firm & $\mathrm{F} 6$ \\
\hline Credit Rating of TSBs & $\mathrm{F} 7$ \\
\hline Past Performance of Firm & $\mathrm{F} 8$ \\
\hline Expertise of Seller Firm & $\mathrm{F} 9$ \\
\hline Promptness in Updating Customer & $\mathrm{F} 10$ \\
\hline Services and Supports & $\mathrm{F} 11$ \\
\hline Resolution of Customer Complaints & $\mathrm{F} 12$ \\
\hline Early Bird Incentives & $\mathrm{F} 13$ \\
\hline Transparency in Information Disclosure & $\mathrm{F} 14$ \\
\hline Compulsory Lock-in Period more than 3 years & $\mathrm{F} 15$ \\
\hline Liquidity in Investment & $\mathrm{F} 16$ \\
\hline
\end{tabular}

Source: Researchers' primary data

\subsection{Descriptive Statistics}

Table 1 represents the descriptive statistics of the data collected.

Table 1 Descriptive Statistics

\begin{tabular}{ccccc}
\hline Attribute & N & Mean & Std. Deviation & Variance \\
\hline \hline F1 & 250 & 1.08 & 0.266 & 0.071 \\
\hline F2 & 250 & 4.89 & 0.398 & 0.158 \\
\hline F3 & 250 & 1.48 & 0.635 & 0.403 \\
\hline F4 & 250 & 4.94 & 0.245 & 0.060 \\
\hline F5 & 250 & 3.84 & 0.860 & 0.740 \\
\hline F6 & 250 & 4.44 & 0.593 & 0.352 \\
\hline F7 & 250 & 4.72 & 0.499 & 0.249 \\
\hline F8 & 250 & 4.76 & 0.428 & 0.183 \\
\hline F9 & 250 & 4.72 & 0.617 & 0.381 \\
\hline F10 & 250 & 4.87 & 0.326 & 0.106 \\
\hline F11 & 250 & 4.73 & 0.444 & 0.197 \\
\hline F12 & 250 & 4.80 & 0.360 & 0.129 \\
\hline F13 & 250 & 3.48 & 0.865 & 0.749 \\
\hline F14 & 250 & 4.73 & 0.446 & 0.199 \\
\hline F15 & 250 & 1.13 & 0.423 & 0.179 \\
\hline F16 & 250 & 3.74 & 0.600 & 0.360 \\
\hline Valid N (listwise) & 250 & & & \\
\hline & S0urce & & & \\
\hline
\end{tabular}

Source: Researchers' Primary Data 
It can be inferred from Table 1 that respondents have responded fairly against each of the questions (attributes). The statistics reveal that the attribute "Tax Benefits from the Investment in TSBS (mean 4.94)" is, obviously, a primary concern to most investors followed by the attribute "Return from the Investment (mean 4.89)" and after sales service quality of the firm i.e. "Promptness in updating customers (mean 4.80)". The attributes "Risk in Investments (mean 1.08)" and "Compulsory Lock-in Period more than 3 years (mean 1.13)" are of least concerns, which is obvious for the reasons that most taxpayers do not want to take any risk while investing in bonds and longer lock-in periods will mean that their money will be stuck in such an investment for a much longer period. Another attribute "Cost of Buying Assets, the TSBs (mean 1.48)" also got negative responses, which may be due to the reason that if cost of buying goes up, the yield on the return comes down.

\subsection{Test of Reliability: Cronbach's Alpha}

In most of the survey-based studies, researchers often use summated scales to probe underlying constructs being measured. These survey instruments may consist of indexed responses to dichotomous or multi point questionnaires, which are later summed to arrive at a resultant score associated with a particular respondent in the study. Usually, developments of such scales are used to gather predictor variables for use in objective models in further researches. Santos (1999) argued that in most such studies, the question is raised on reliability. As the function of scales is to encompass the realm of prediction stretches, the reliability of data consistency becomes an issue. One of the most popular reliability statistics in use in such studies is Cronbach's alpha (Cronbach, 1951).

Cronbach's alpha is a measure of internal consistency (i.e. how closely related a set of items are as a group). As a rule of thumb, a high value of alpha (of 0.70 or more) is often used as evidence that the items measure an underlying (or latent) construct, meaning a high degree of consistency. In the present study, Cronbach's Alpha is found to be 0.889 , which sufficiently meets the minimum requirement to move ahead in the study (for other tests such as KMO, Barlett's and Factor Analysis).

\subsection{Kaiser-Meyer-Olkin Test of Adequacy and Bartlett's Test of Sphericity}

The KMO test is a measure of sampling adequacy which compares the magnitudes of the observed correlation coefficients in relation to the magnitudes of the partial correlation coefficients. A partial correlation measures the strength of the relationship between any two variables (or attributes) when the other variables (attributes) are kept constant. Generally, a large KMO value is considered a good measure which agrees that the correlations between pairs of variables (i.e. potential factors or attributes) can be explained by the other variables (or 
attributes). If the $\mathrm{KMO}$ test results in a measure equal or below 0.5 , then factor analysis cannot be performed.

Barlett's test of sphericity (Barlett, 1950) is a test for the hypothesis that the correlation matrix is an identity matrix (i.e. all the diagonal terms are 1 and nondiagonal terms are zero). Basically, before applying factor analysis, what to be determined first is whether the hypothesis that all the correlations, tested simultaneously, are zero or significantly different from zero. In case correlations are significantly different from zero can be rejected. To make it simple to understand, imagine a correlation matrix where all variables (attributes) are perfectly correlated (=1) with oneself, and have some level of correlation with the other variables (attributes). If the variables are not correlated with the other variables then these variables can't be part of the same factor.

Table 2 represents the results of Bartlett's and KMO tests. The KMO measure of sample adequacy is 0.837 , which can be considered of higher order and a good score to continue. Bartlett's tests of sphericity results in a significant level of < 0.01 which is fulfilling the minimum requirement of $\leq 0.05$ and a clear indication that factor analysis can be applied on the data.

Table $2 \mathrm{KMO}$ and Bartlett's Test

\begin{tabular}{l|l|c}
\hline \multicolumn{2}{l}{ Kaiser-Meyer-Olkin Measure of Sampling Adequacy. } & $\mathbf{0 . 8 3 7}$ \\
\hline \multirow{3}{*}{ Bartlett's Test of Sphericity } & Approx. Chi-Square & 650.251 \\
\cline { 2 - 3 } & DF & 120 \\
\cline { 2 - 3 } & Sig. & $\mathbf{0 . 0 0 0}$ \\
\hline
\end{tabular}

Source: Researchers' Primary Data

\subsection{Correlation Analysis: Test of Multicollinearity}

Further, the correlation analysis is performed on the data to study the problem of multicollinearity, which exists when two or more of the predictors in a regression model are moderately or highly correlated. Correlation is a measure of the association between two variables. It indicates how the value of one variable changes with the changes in the value of the other correlated variable. A correlation coefficient of zero means that there is no association between the variables while a correlation coefficient of -1 would mean a perfectly inverse correlation between variables i.e. variable will move in opposite directions with same magnitude of changes. A correlation coefficient of +1 would mean that correlated variables would move with same magnitude of positive changes. The problem of multicollinearity is assumed to exist if the correlation coefficient between two or more variables under tests is more than 0.5 and if so, the data would require factor analysis to be performed. Table 3 represents the correlation matrix. It can be inferred from the results that correlation coefficient between some variables is more than 0.5 which, therefore, requires factor analysis to be performed on the data. 
Table 3 Correlation Matrix (Source: Researcher's Primary Data)

\begin{tabular}{|c|c|c|c|c|c|c|c|c|c|c|c|c|c|c|c|c|}
\hline & it & $\mathbf{N}$ & $\underline{m}$ & $\mathbb{4}$ & 눈 & டீ & is & $\stackrel{\infty}{\mathbb{4}}$ & 욘 & $\underset{\text { 언 }}{0}$ & $\vec{t}$ & $\underset{\sim}{\mathbf{H}}$ & $\underset{H}{M}$ & $\underset{i}{ \pm}$ & $\stackrel{\underline{n}}{\sim}$ & $\begin{array}{l}0 \\
\underline{-1} \\
\underline{4}\end{array}$ \\
\hline iㅏㄴ & $\begin{array}{l}8 \\
8 \\
\circ \\
-1\end{array}$ & $\begin{array}{l}0 \\
\dot{0} \\
0 \\
1\end{array}$ & $\begin{array}{l}\text { O } \\
\stackrel{+}{+} \\
\text { - }\end{array}$ & $\begin{array}{l}0 \\
\text { ㄱ. } \\
\text { : } \\
1\end{array}$ & $\begin{array}{l}0 \\
0 \\
0 \\
0 \\
1 \\
1\end{array}$ & $\begin{array}{l}N \\
0 \\
-1 \\
0 \\
1\end{array}$ & $\begin{array}{l}\underset{J}{J} \\
\stackrel{0}{0} \\
i\end{array}$ & $\begin{array}{l}0 \\
0 \\
0 \\
0 \\
1 \\
1\end{array}$ & $\begin{array}{l}m \\
\text { - } \\
-1 \\
0 \\
1\end{array}$ & $\begin{array}{l}0 \\
\infty \\
0 \\
0 \\
1 \\
1\end{array}$ & $\begin{array}{l}\text { Ln } \\
\text { ○ } \\
\\
0\end{array}$ & $\begin{array}{l}\hat{m} \\
0 \\
0\end{array}$ & $\begin{array}{l}9 \\
\stackrel{-}{-1} \\
-1 \\
0\end{array}$ & $\begin{array}{l}\text { 今 } \\
\text { - } \\
\text { - }\end{array}$ & 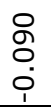 & $\begin{array}{l}\text { N } \\
\text { o } \\
0\end{array}$ \\
\hline$\underset{\mathbf{N}}{\mathbf{N}}$ & $\begin{array}{l}0 \\
\dot{+} \\
0 \\
0 \\
1\end{array}$ & $\begin{array}{l}8 \\
\text { ○ } \\
\text { - }\end{array}$ & $\begin{array}{l}\text { nn } \\
\text { م⿱ } \\
0 \\
0 \\
\end{array}$ & $\begin{array}{l}\hat{A} \\
0 \\
0\end{array}$ & $\begin{array}{l}\text { Na } \\
0 \\
0 \\
1\end{array}$ & 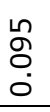 & $\begin{array}{l}\stackrel{0}{\circ} \\
0 \\
0\end{array}$ & $\begin{array}{l}\text { Mn } \\
\text { ஸी } \\
0 \\
0\end{array}$ & $\begin{array}{l}\text { 고 } \\
\circ \\
0\end{array}$ & $\begin{array}{l}\stackrel{\infty}{\sim} \\
\underset{0}{-} \\
0\end{array}$ & $\begin{array}{l}m \\
0 \\
0 \\
\dot{0}\end{array}$ & $\begin{array}{l}m \\
\stackrel{m}{0} \\
0 \\
\dot{1}\end{array}$ & \begin{tabular}{l}
$m$ \\
\multirow{\Delta}{0}{} \\
$\dot{0}$
\end{tabular} & $\begin{array}{l}\text { m̂n } \\
\text { م⿱ } \\
0 \\
1\end{array}$ & 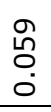 & $\begin{array}{l}\infty \\
\stackrel{8}{0} \\
0 \\
0\end{array}$ \\
\hline$\underset{u}{m}$ & $\begin{array}{l}\stackrel{0}{+} \\
\stackrel{-}{0} \\
0\end{array}$ & $\begin{array}{l}\text { Ln } \\
\text { مِ } \\
0 \\
0 \\
1\end{array}$ & $\begin{array}{l}8 \\
8 \\
\end{array}$ & 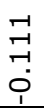 & $\begin{array}{l}\text { O } \\
\text { ல } \\
0\end{array}$ & 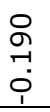 & $\begin{array}{l}\text { กิ } \\
0 \\
0\end{array}$ & 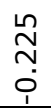 & $\begin{array}{l}\text { m } \\
\text { न1 } \\
\text { i } \\
1\end{array}$ & $\begin{array}{l}\underset{\infty}{\infty} \\
\stackrel{1}{N} \\
\vdots \\
1\end{array}$ & $\stackrel{\stackrel{L}{+}}{\stackrel{0}{0}}$ & $\begin{array}{l}m \\
0 \\
0 \\
0 \\
1\end{array}$ & $\begin{array}{l}0 \\
\text { mo } \\
0 \\
0\end{array}$ & 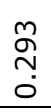 & $\begin{array}{l}\tilde{m} \\
0 \\
0 \\
0\end{array}$ & $\begin{array}{l}\stackrel{m}{二} \\
\stackrel{-}{0} \\
0\end{array}$ \\
\hline 妾 & $\begin{array}{l}0 \\
-1 \\
-1 \\
-1 \\
1 \\
1\end{array}$ & $\begin{array}{l}\hat{A} \\
\text { ○ } \\
0\end{array}$ & 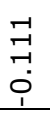 & $\begin{array}{l}\circ \\
\text { ○ } \\
\text { - }\end{array}$ & \begin{tabular}{l}
\multirow{0}{0}{} \\
0 \\
0 \\
0
\end{tabular} & $\begin{array}{l}\text { बे } \\
\text { '- } \\
0\end{array}$ & $\begin{array}{l}\text { กิ } \\
\circ \\
0 \\
0\end{array}$ & 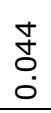 & $\begin{array}{l}\text { N } \\
\text { ○े } \\
0\end{array}$ & 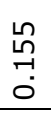 & $\begin{array}{l}\stackrel{-}{N} \\
\stackrel{-}{0} \\
i\end{array}$ & $\begin{array}{l}-7 \\
\text { - } \\
\overrightarrow{1} \\
1 \\
1\end{array}$ & $\begin{array}{l}\hat{m} \\
\stackrel{-}{0} \\
i \\
1\end{array}$ & $\begin{array}{l}\underset{N}{N} \\
\underset{\sim}{0} \\
i\end{array}$ & $\begin{array}{l}\text { ナ } \\
\circ \\
\circ \\
\end{array}$ & \begin{tabular}{l}
\multirow{2}{*}{} \\
$\underset{-}{-}$ \\
0 \\
1 \\
1
\end{tabular} \\
\hline 눈 & $\begin{array}{l}0 \\
-1 \\
0 \\
0 \\
1\end{array}$ & $\begin{array}{l}\text { Na } \\
0 \\
0 \\
1\end{array}$ & $\begin{array}{l}g \\
\text { ப } \\
0 \\
0\end{array}$ & \begin{tabular}{l}
\multirow{\Xi}{}{} \\
$\vdots$ \\
$\vdots$
\end{tabular} & $\begin{array}{l}8 \\
\text { ○ } \\
\text { - }\end{array}$ & $\begin{array}{l}\text { ભ̊ } \\
0 \\
0\end{array}$ & 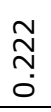 & $\begin{array}{l}\stackrel{J}{\mathbb{N}} \\
0 \\
0\end{array}$ & $\begin{array}{l}\text { mે } \\
\text { ○े } \\
\stackrel{0}{0}\end{array}$ & $\begin{array}{l}\text { Ln } \\
\infty \\
0 \\
0 \\
1\end{array}$ & $\begin{array}{l}\text { M̂ } \\
\text { ஸ̂ } \\
0\end{array}$ & 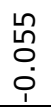 & 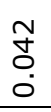 & $\begin{array}{l}\stackrel{\text { n}}{m} \\
\stackrel{-}{0} \\
0\end{array}$ & 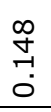 & \begin{tabular}{l}
\multirow{2}{*}{} \\
0 \\
0 \\
0 \\
1
\end{tabular} \\
\hline 운 & $\begin{array}{l}0 \\
0 \\
-1 \\
0 \\
1\end{array}$ & $\begin{array}{l}\text { Ln } \\
\text { ọ } \\
\dot{0}\end{array}$ & $\begin{array}{l}\text { ᄋ } \\
\text {-1 } \\
\text { i. }\end{array}$ & 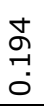 & $\begin{array}{l}\text { M̂ } \\
\varnothing \\
0\end{array}$ & $\begin{array}{l}8 \\
8 \\
\end{array}$ & 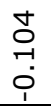 & $\underset{\sim}{\sim}$ & 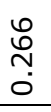 & 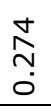 & $\begin{array}{l}m \\
\infty \\
\stackrel{\infty}{N} \\
\vdots \\
1\end{array}$ & $\begin{array}{l}\text { L } \\
8 \\
0 \\
\dot{1}\end{array}$ & $\begin{array}{l}\hat{n} \\
\circ \\
0 \\
i\end{array}$ & $\begin{array}{l}\stackrel{J}{ \pm} \\
\stackrel{1}{0} \\
i\end{array}$ & 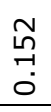 & $\begin{array}{l}\infty \\
\infty \\
0 \\
0 \\
0 \\
1\end{array}$ \\
\hline 는 & \begin{tabular}{l}
8 \\
\multirow{J}{*}{} \\
0 \\
1 \\
1
\end{tabular} & 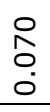 & $\begin{array}{l}\text { N̂. } \\
0 \\
0\end{array}$ & $\begin{array}{l}\text { ஸू } \\
\text { ஸุ } \\
0\end{array}$ & $\underset{N}{N}$ & $\begin{array}{l}\text { J } \\
\text { - } \\
\text { o. } \\
\text { I }\end{array}$ & $\begin{array}{l}8 \\
\text { ¿ } \\
\end{array}$ & \begin{tabular}{l}
\multirow{N}{*}{} \\
$\stackrel{-}{0}$ \\
$\stackrel{1}{1}$
\end{tabular} & $\begin{array}{l}0 \\
\stackrel{1}{N} \\
\\
1\end{array}$ & $\begin{array}{l}\text { n } \\
\stackrel{2}{n} \\
\text { o } \\
1\end{array}$ & $\begin{array}{l}\infty \\
m \\
m \\
0\end{array}$ & 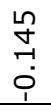 & $\begin{array}{l}\text { I } \\
\text {-1. } \\
\text { i }\end{array}$ & $\begin{array}{l}\overrightarrow{1} \\
m \\
\dot{m} \\
0\end{array}$ & $\begin{array}{l}0 \\
\stackrel{-}{1} \\
\overrightarrow{0} \\
\end{array}$ & $\begin{array}{l}8 \\
8 \\
0 \\
0 \\
1\end{array}$ \\
\hline 悉 & $\begin{array}{l}0 \\
0 \\
0 \\
0 \\
1 \\
1\end{array}$ & $\begin{array}{l}\text { m̃ } \\
\text { ஸ̊ } \\
0\end{array}$ & \begin{tabular}{l} 
N \\
\multirow{N}{*}{} \\
$\stackrel{1}{1}$
\end{tabular} & 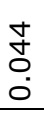 & $\begin{array}{l}\stackrel{+}{N} \\
0 \\
0\end{array}$ & $\begin{array}{l}\stackrel{N}{\sim} \\
\underset{0}{ }\end{array}$ & $\begin{array}{l}\text { N } \\
\stackrel{+}{*} \\
\stackrel{1}{1}\end{array}$ & $\begin{array}{l}8 \\
\text { ¿ } \\
\end{array}$ & $\begin{array}{l}\stackrel{+}{m} \\
\text { m. } \\
0\end{array}$ & 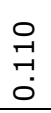 & 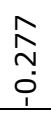 & \begin{tabular}{l}
9 \\
I \\
\hdashline \\
0
\end{tabular} & $\begin{array}{l}\stackrel{J}{ } \\
\\
0\end{array}$ & $\begin{array}{l}\stackrel{\infty}{N} \\
\stackrel{N}{0} \\
i\end{array}$ & $\begin{array}{l}0 \\
\stackrel{1}{-} \\
\stackrel{0}{0}\end{array}$ & $\begin{array}{l}\mathscr{0} \\
0 \\
-1 \\
0 \\
0\end{array}$ \\
\hline 욘 & $\begin{array}{l}m \\
-1 \\
-1 \\
0 \\
1\end{array}$ & $\begin{array}{l}-1 \\
\circ \\
0\end{array}$ & $\begin{array}{l}m \\
\text { I- } \\
\text { o. } \\
1 \\
1\end{array}$ & $\begin{array}{l}\text { ने } \\
\circ \\
0\end{array}$ & $\begin{array}{l}m \\
m \\
0 \\
0\end{array}$ & $\begin{array}{l}\mathscr{\bullet} \\
\stackrel{2}{N} \\
0\end{array}$ & $\begin{array}{l}\omega \\
\stackrel{2}{N} \\
0 \\
1 \\
1\end{array}$ & $\begin{array}{l}\text { mे } \\
\text { m. } \\
0\end{array}$ & $\begin{array}{l}8 \\
8 \\
-i\end{array}$ & 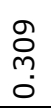 & 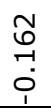 & $\begin{array}{l}\text { J } \\
\circ \\
0 \\
0\end{array}$ & 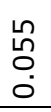 & $\begin{array}{l}\text { oे } \\
\text { N̦} \\
\text { o } \\
\end{array}$ & $\begin{array}{l}\text { Iิ } \\
\text { - } \\
0\end{array}$ & $\begin{array}{l}\hat{0} \\
\text { - } \\
\text { - } \\
0\end{array}$ \\
\hline 욱 & $\begin{array}{l}0 \\
0 \\
0 \\
0 \\
1 \\
1\end{array}$ & $\begin{array}{l}\stackrel{\infty}{\sim} \\
\stackrel{-}{0} \\
\end{array}$ & \begin{tabular}{l}
\multirow{\infty}{*}{} \\
N̦ \\
i
\end{tabular} & $\begin{array}{l}\stackrel{\text { nn }}{n} \\
\stackrel{-}{0} \\
0\end{array}$ & $\begin{array}{l}n \\
\infty \\
0 \\
0 \\
0 \\
1\end{array}$ & 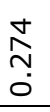 & $\begin{array}{l}\text { Ln } \\
\text { N̦} \\
\text { i } \\
1\end{array}$ & $\begin{array}{l}\circ \\
\stackrel{0}{-1} \\
\\
0\end{array}$ & $\begin{array}{l}\text { gे } \\
\text { mे } \\
\text { o. }\end{array}$ & $\begin{array}{l}8 \\
\circ \\
\\
\end{array}$ & $\begin{array}{l}\infty \\
0 \\
-1 \\
0 \\
1 \\
1\end{array}$ & $\begin{array}{l}m \\
ٌ \\
0 \\
0 \\
1 \\
1\end{array}$ & $\begin{array}{l}0 \\
\tilde{n} \\
0 \\
0\end{array}$ & 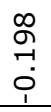 & $\begin{array}{l}\text { hે } \\
\text { ஸे } \\
0\end{array}$ & $\begin{array}{l}n \\
1 \\
0 \\
0 \\
0 \\
1 \\
1\end{array}$ \\
\hline$\vec{H}$ & $\begin{array}{l}\text { ñ } \\
\stackrel{0}{1} \\
0 \\
0\end{array}$ & $\begin{array}{l}m \\
0 \\
0 \\
0 \\
1\end{array}$ & \begin{tabular}{l}
$\stackrel{n}{N}$ \\
\multirow{2}{0}{} \\
$\vdots$
\end{tabular} & \begin{tabular}{l} 
İ \\
\multirow{1}{*}{} \\
i.
\end{tabular} & $\begin{array}{l}\text { Mn } \\
\text { م̊ } \\
0\end{array}$ & $\begin{array}{l}m \\
\infty \\
N \\
0 \\
1\end{array}$ & $\begin{array}{l}\infty \\
\stackrel{\infty}{m} \\
m \\
0\end{array}$ & 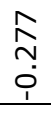 & 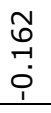 & $\begin{array}{l}\infty \\
0 \\
-1 \\
0 \\
1 \\
1\end{array}$ & ঃ & $\begin{array}{l}\text { Ln } \\
\circ \\
0 \\
0 \\
1\end{array}$ & $\begin{array}{l}\vec{m} \\
\overrightarrow{1} \\
\stackrel{0}{1}\end{array}$ & $\begin{array}{l}\text { Ln } \\
\mathscr{0} \\
\ddot{0}\end{array}$ & $\begin{array}{l}\infty \\
\stackrel{1}{-1} \\
\stackrel{0}{0} \\
0\end{array}$ & 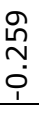 \\
\hline$\underset{\sim}{\stackrel{N}{4}}$ & $\begin{array}{l}\hat{m} \\
0 \\
0\end{array}$ & $\begin{array}{l}m \\
\stackrel{1}{0} \\
0 \\
1\end{array}$ & $\begin{array}{l}m \\
\stackrel{-}{0} \\
0 \\
1 \\
1\end{array}$ & $\begin{array}{l}-7 \\
\text {-1 } \\
\stackrel{0}{1} \\
1\end{array}$ & $\begin{array}{l}\text { Ln } \\
\text { ○ } \\
0 \\
1 \\
1\end{array}$ & $\begin{array}{l}\text { L } \\
\circ \\
0 \\
\\
1\end{array}$ & 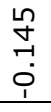 & $\begin{array}{l}9 \\
\stackrel{-}{-} \\
0\end{array}$ & $\begin{array}{l}\text { मे } \\
\text { ठ } \\
\text { ठ }\end{array}$ & $\begin{array}{l}\text { ñ } \\
\circ \\
0 \\
1\end{array}$ & $\begin{array}{l}\text { Ln } \\
\circ \\
0 \\
0 \\
1\end{array}$ & $\begin{array}{l}8 \\
\text { ¿ } \\
\end{array}$ & 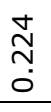 & $\begin{array}{l}\text { I- } \\
\text { न- } \\
0\end{array}$ & $\begin{array}{l}0 \\
0 \\
ت \\
0\end{array}$ & $\begin{array}{l}\underset{\infty}{N} \\
\underset{0}{0}\end{array}$ \\
\hline$\underset{\vec{L}}{\stackrel{m}{H}}$ & $\begin{array}{l}\text { G } \\
-1 \\
-1 \\
0\end{array}$ & \begin{tabular}{l}
$m$ \\
\multirow{O}{*}{} \\
$\dot{0}$
\end{tabular} & $\begin{array}{l}\stackrel{0}{ } \\
\text { o } \\
0 \\
0\end{array}$ & $\begin{array}{l}\hat{m} \\
\stackrel{1}{0} \\
i \\
1\end{array}$ & 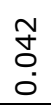 & $\begin{array}{l}\hat{n} \\
0 \\
0 \\
\text { i }\end{array}$ & \begin{tabular}{l} 
I \\
\multirow{1}{*}{} \\
$i$ \\
1
\end{tabular} & $\begin{array}{l}\text { J } \\
\text { O } \\
0\end{array}$ & 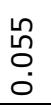 & $\begin{array}{l}0 \\
\stackrel{0}{0} \\
0\end{array}$ & $\begin{array}{c}\vec{m} \\
\stackrel{-}{0} \\
i \\
1\end{array}$ & $\begin{array}{l}\stackrel{J}{N} \\
\underset{0}{0}\end{array}$ & $\begin{array}{l}\circ \\
\circ \\
\end{array}$ & 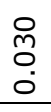 & $\begin{array}{l}\circ \\
\stackrel{-}{-} \\
\stackrel{-}{0} \\
\end{array}$ & $\begin{array}{l}\infty \\
\vec{\sigma} \\
\vec{\sigma} \\
0\end{array}$ \\
\hline$\underset{\mathbf{u}}{\mathbb{H}}$ & $\begin{array}{l}\hat{0} \\
\text { - } \\
0 \\
0\end{array}$ & $\begin{array}{l}\text { ma } \\
\text { ڤొ } \\
0 \\
1\end{array}$ & $\begin{array}{l}\text { @ે } \\
\text { స్} \\
\vdots\end{array}$ & 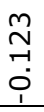 & $\begin{array}{l}\stackrel{n}{m} \\
\stackrel{-}{-} \\
0\end{array}$ & 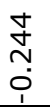 & $\begin{array}{l}\overrightarrow{-} \\
\vec{m} \\
\stackrel{0}{0}\end{array}$ & 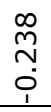 & $\begin{array}{l}\text { Oे } \\
\text { N̦} \\
\text { i }\end{array}$ & $\begin{array}{l}\infty \\
0 \\
\\
0 \\
1\end{array}$ & $\begin{array}{l}\text { Ln } \\
\mathscr{0} \\
0 \\
0\end{array}$ & $\begin{array}{l}\text { 근 } \\
\text { न- } \\
0\end{array}$ & $\begin{array}{l}0 \\
\text { m } \\
0 \\
0\end{array}$ & $\begin{array}{l}8 \\
8 \\
\end{array}$ & $\begin{array}{l}\overrightarrow{-} \\
\text { ने } \\
0\end{array}$ & $\begin{array}{l}\text { Oे } \\
\text { ஸे } \\
0 \\
0\end{array}$ \\
\hline$\underset{\mathbf{L}}{\mathbf{H}}$ & $\begin{array}{l}8 \\
8 \\
0 \\
0 \\
1\end{array}$ & $\begin{array}{l}\text { ํ. } \\
\circ \\
0 \\
0\end{array}$ & $\begin{array}{l}N \\
\\
0 \\
0\end{array}$ & 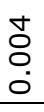 & $\begin{array}{l}\infty \\
\stackrel{+}{+} \\
0\end{array}$ & $\begin{array}{l}\text { N̂} \\
\text { - } \\
0 \\
0\end{array}$ & $\begin{array}{l}0 \\
⿱ \\
-1 \\
0 \\
0\end{array}$ & $\begin{array}{l}0 \\
\stackrel{1}{7} \\
0 \\
0\end{array}$ & $\begin{array}{l}\text { ్ } \\
\text { - } \\
0\end{array}$ & $\begin{array}{l}\hat{n} \\
\circ \\
0\end{array}$ & $\begin{array}{l}\infty \\
\stackrel{1}{-1} \\
\stackrel{0}{0} \\
\end{array}$ & $\begin{array}{l}\qquad \\
\stackrel{-}{-1} \\
\vdots \\
\end{array}$ & $\begin{array}{l}0 \\
\stackrel{-}{-1} \\
\stackrel{-}{0}\end{array}$ & $\begin{array}{l}\overrightarrow{-} \\
\stackrel{-}{-1} \\
\dot{0}\end{array}$ & $\begin{array}{l}8 \\
\text { ○ } \\
\text { - }\end{array}$ & $\begin{array}{l}\stackrel{+}{m} \\
\vec{\sim} \\
0\end{array}$ \\
\hline $\begin{array}{l}\underset{-1}{L} \\
\vec{L}\end{array}$ & 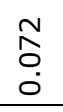 & $\begin{array}{l}\infty \\
\stackrel{0}{0} \\
0\end{array}$ & $\begin{array}{l}\stackrel{m}{-} \\
\underset{-1}{0} \\
0\end{array}$ & $\begin{array}{l}\stackrel{7}{7} \\
\stackrel{-}{0} \\
\stackrel{1}{1}\end{array}$ & $\begin{array}{l}\text { Na } \\
0 \\
0 \\
1\end{array}$ & $\begin{array}{l}\infty \\
\infty \\
0 \\
0 \\
0 \\
1\end{array}$ & $\begin{array}{l}8 \\
8 \\
0 \\
1 \\
1\end{array}$ & $\begin{array}{l}0 \\
0 \\
-1 \\
0\end{array}$ & $\begin{array}{l}\text { 今. } \\
\text { ㄱ. } \\
\text { ○. }\end{array}$ & 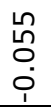 & 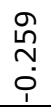 & $\begin{array}{l}\stackrel{\infty}{N} \\
\underset{0}{0} \\
\end{array}$ & $\begin{array}{l}\infty \\
\stackrel{\infty}{*} \\
\vec{\sigma} \\
0\end{array}$ & $\begin{array}{l}\sigma \\
\text { o } \\
0 \\
0\end{array}$ & $\begin{array}{l}\stackrel{+}{m} \\
\stackrel{-}{0} \\
\end{array}$ & $\begin{array}{l}8 \\
\stackrel{8}{ } \\
\end{array}$ \\
\hline
\end{tabular}




\section{Factor Analysis}

In a questionnaire based survey research, the responses to each question (the variable or attribute) represent an outcome. But sometimes many questions in the survey are often related, this might tend to influence subject responses. Factor analysis explains correlations among multiple outcomes as the result of one or more underlying explanations, or factors. The process involves data reduction, as it attempts to represent a set of variables by a smaller number. Factor analysis takes qualitative observations against each of the variables and resolves them into distinct patterns of occurrence. In other words, factor analysis reduces the large number of variables in the study to a smaller number that is capable of explaining the same variance observed in the large number of variables.

\subsection{Communalities}

Communality measures the extent to which a variable correlates with all other variables and higher values of communalities are considered better. If communalities for a particular variable are low (say, between 0.0 and 0.5 ), then that variable will struggle to load significantly on any factor. Once the extraction of factors has been completed, the table of communalities is examined for how much of the variance in each of the original variables is explained by the extracted factors. Table 4 represents the results on communalities of variables (the amount of variation extracted from each variable). In the present study, the extraction of factors is done using the principal component analysis method.

From the table below, it can be inferred that variables "Service and Support $(0.824)$ ", "Transparency in information disclosure $(0.781)$ ", and "Return from investment $(0.714)$ " carried the highest communalities. The table also shows communalities for three variables below 0.50, "Risk in investment (0.281)", "Cost of buying Asset (0.321)", and "Fringe Benefits (0.471)". The communalities less than 0.5 would mean that these variables are not explained adequately by the factors i.e. such variables do not correspond sufficiently to any of the factors and could thus be discarded.

Table 4 Communalities

\begin{tabular}{ccc}
\hline Attribute & Initial & Extraction \\
\hline \hline F1 & 1.000 & 0.281 \\
\hline F2 & 1.000 & 0.714 \\
\hline F3 & 1.000 & 0.321 \\
\hline F4 & 1.000 & 0.596 \\
\hline F5 & 1.000 & 0.471 \\
\hline F6 & 1.000 & 0.610 \\
\hline F7 & 1.000 & 0.630 \\
\hline F8 & 1.000 & 0.558 \\
\hline
\end{tabular}




\begin{tabular}{ccc}
\hline Attribute & Initial & Extraction \\
\hline \hline F9 & 1.000 & 0.553 \\
\hline F10 & 1.000 & 0.568 \\
\hline F11 & 1.000 & 0.824 \\
\hline F12 & 1.000 & 0.525 \\
\hline F13 & 1.000 & 0.581 \\
\hline F14 & 1.000 & 0.781 \\
\hline F15 & 1.000 & 0.525 \\
\hline F16 & 1.000 & 0.674 \\
\hline
\end{tabular}

Extraction Method: Principal Component Analysis.

Source: Researchers' Primary Data

\subsection{Total Variance Explained}

All the variables in the study are further analyzed for Eigenvalues. The Eigenvalue for a given factor measures the variance in all the variables which is accounted for by that factor. In other words, the Eigenvalue is the total variance explained by each factor. Any factor that has an Eigenvalue less than one would be disregarded because this factor does not have enough total variance explained to represent a unique factor.

The un-rotated output in factor analysis tends to maximize the variance accounted for by the first and subsequent factors, thus forcing the factors to be orthogonal in factor matrix. During this stage of data compression, most variables tend to load on the early factors and then substantially on more than one factor. Here, rotation serves a better option by making output more understandable by generating a pattern of loadings where variables load strongly on one factor while loading weakly on the other subsequent factors. Rotations of loading can be either orthogonal or oblique rotation, allowing the factors to correlate.

The most commonly used method of orthogonal rotation is Varimax Rotation, which rotates the factor axes to maximize the variance of the loadings of a factor in column on all the variables in rows of a factor matrix (representing the results of differentiating the original variables by extracted factors). However, often unrealistic assumption of orthogonality causes the Varimax Rotation to be a less preferred method. The other option of factor rotation, Oblique method, which is inclusive of orthogonal rotation, is rather more preferred method in factor analysis. But in this study, the Varimax Rotation method is used.

Table 5 (below) represents the results in total variance explained by different variables in the study. The extraction is done through the principal component analysis and orthogonal (varimax) rotation method of rotation for factor loading. 
Table 5 Total Variance Explained By Different Variables

\begin{tabular}{|c|c|c|c|c|c|c|c|}
\hline \multirow{2}{*}{ 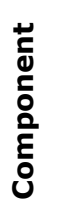 } & \multicolumn{3}{|c|}{ Initial Eigenvalues } & \multicolumn{3}{|c|}{$\begin{array}{c}\text { Extraction Sums of Squared } \\
\text { Loadings }\end{array}$} & \multirow{2}{*}{$\begin{array}{c}\begin{array}{c}\text { Rotation Sums } \\
\text { of Squared } \\
\text { Loadings }^{\mathrm{a}}\end{array} \\
\text { Total }\end{array}$} \\
\hline & Total & $\begin{array}{c}\% \text { of } \\
\text { Variance }\end{array}$ & $\begin{array}{c}\text { Cumulative } \\
\%\end{array}$ & Total & $\begin{array}{c}\% \text { of } \\
\text { Variance }\end{array}$ & $\underset{\%}{\text { Cumulative }}$ & \\
\hline 1 & 2.834 & 17.711 & 17.711 & 2.834 & 17.711 & 17.711 & 2.219 \\
\hline 2 & 1.914 & 11.960 & 29.671 & 1.914 & 11.960 & 29.671 & 1.848 \\
\hline 3 & 1.593 & 9.958 & 39.629 & 1.593 & 9.958 & 39.629 & 1.484 \\
\hline 4 & 1.148 & 7.175 & 46.805 & 1.148 & 7.175 & 46.805 & 2.247 \\
\hline 5 & 1.062 & 6.639 & 53.443 & 1.062 & 6.639 & 53.443 & 1.360 \\
\hline 6 & 0.990 & 6.190 & 59.633 & & & & \\
\hline 7 & 0.897 & 5.604 & 65.237 & & & & \\
\hline 8 & 0.860 & 5.376 & 70.613 & & & & \\
\hline 9 & 0.835 & 5.221 & 75.834 & & & & \\
\hline 10 & 0.772 & 4.824 & 80.659 & & & & \\
\hline 11 & 0.752 & 4.698 & 85.357 & & & & \\
\hline 12 & 0.619 & 3.870 & 89.226 & & & & \\
\hline 13 & 0.543 & 3.391 & 92.618 & & & & \\
\hline 14 & 0.506 & 3.165 & 95.782 & & & & \\
\hline 15 & 0.439 & 2.743 & 98.525 & & & & \\
\hline 16 & 0.236 & 1.475 & 100.000 & & & & \\
\hline
\end{tabular}

Extraction Method: Principal Component Analysis.

a. When components are correlated, sums of squared loadings cannot be added to obtain a total variance.

Source: Researchers' Primary Data

From the results above in table 5, it is clearly evident that there are five variables which have Eigenvalues more than 1.0 and the cumulative total variance explained by these factors is $53.44 \%$. The sorted Eigenvalue against the factor number is graphed in the Cattell Scree Plot in the picture below (Picture 1). The plot reckons like the side of a mountain and Scree refers to the debris falling from that mountain and settling at the mountain's base.

The graph below exhibits that there are five factors which are more important to taxpayers (with Eigenvalues either equal or more than 1). The rest of the factors, though also influenced the investors in some ways or the other, are on limited scale (below Eigenvalue 1). 
Picture 1 Screen Plot

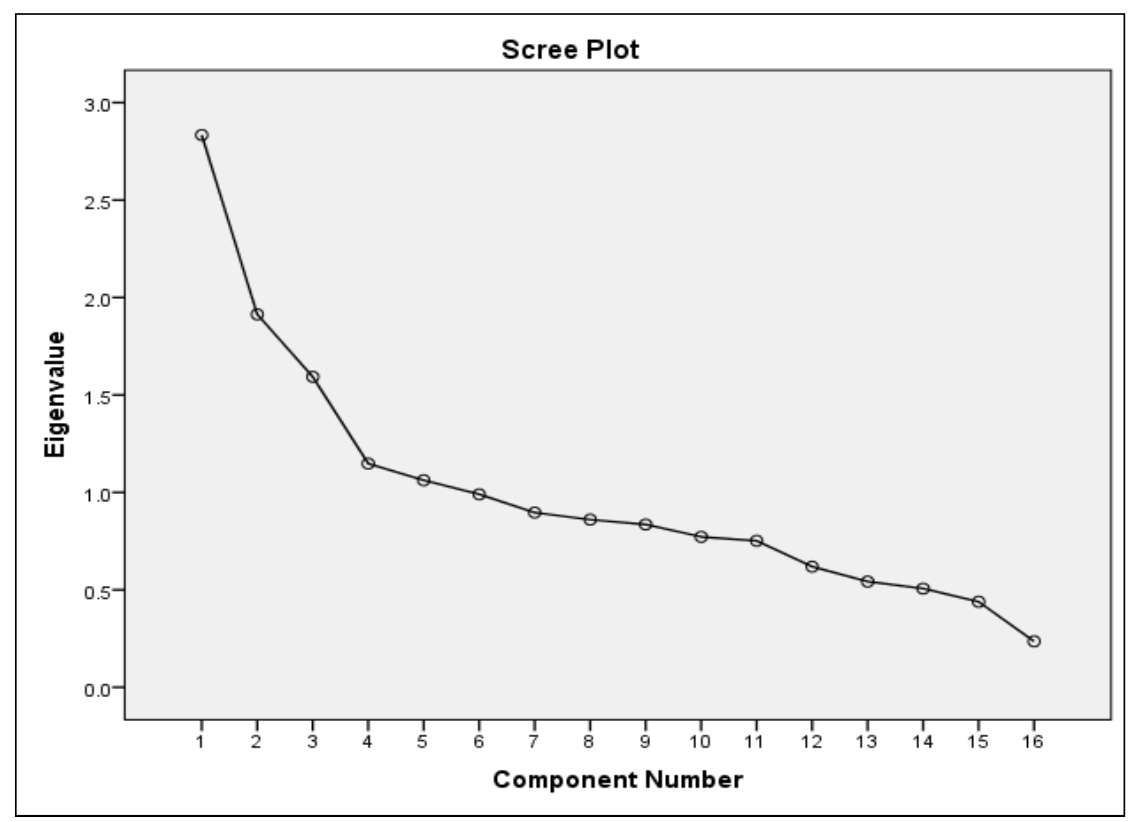

Source: Researchers' Primary Data

\subsection{Component Matrix}

The component matrix shown in Table 6 below indicates how each variable in the analysis correlates with each of the five retained factors. The same weight is carried by both negative and positive correlations.

Table 6 Component Matrix ${ }^{a}$

\begin{tabular}{c|c|c|c|c|c}
\hline \multirow{2}{*}{ Attribute } & \multicolumn{5}{|c}{ Component } \\
\cline { 2 - 6 } & $\mathbf{1}$ & $\mathbf{2}$ & $\mathbf{3}$ & $\mathbf{4}$ & $\mathbf{5}$ \\
\hline \hline F1 & -0.721 & -0.121 & 0.228 & 0.484 & 0.051 \\
\hline F2 & -0.711 & 0.167 & 0.374 & 0.327 & 0.031 \\
\hline F3 & 0.562 & 0.128 & 0.241 & 0.347 & -0.207 \\
\hline F4 & 0.549 & -0.199 & 0.319 & 0.053 & -0.062 \\
\hline F5 & 0.530 & 0.250 & 0.228 & 0.046 & -0.244 \\
\hline F6 & 0.524 & -0.155 & 0.110 & 0.394 & 0.320 \\
\hline F7 & -0.508 & 0.226 & -0.039 & -0.075 & -0.069 \\
\hline F8 & 0.107 & 0.723 & 0.075 & -0.347 & 0.118 \\
\hline F9 & 0.089 & 0.684 & 0.026 & -0.151 & 0.286 \\
\hline F10 & 0.072 & 0.567 & 0.092 & 0.293 & -0.057 \\
\hline F11 & 0.252 & -0.393 & 0.227 & -0.147 & 0.067 \\
\hline F12 & 0.055 & 0.249 & 0.672 & 0.078 & -0.051 \\
\hline
\end{tabular}




\begin{tabular}{c|c|c|c|c|c}
\hline \multirow{2}{*}{ Attribute } & \multicolumn{5}{|c}{ Component } \\
\cline { 2 - 6 } & $\mathbf{1}$ & $\mathbf{2}$ & $\mathbf{3}$ & $\mathbf{4}$ & $\mathbf{5}$ \\
\hline \hline $\mathrm{F} 13$ & -0.118 & -0.017 & 0.511 & -0.320 & -0.305 \\
\hline $\mathrm{F} 14$ & -0.464 & -0.230 & 0.484 & -0.345 & 0.092 \\
\hline $\mathrm{F} 15$ & -0.204 & 0.302 & -0.328 & 0.200 & 0.013 \\
\hline F16 & 0.120 & -0.070 & 0.235 & -0.041 & 0.798 \\
\hline
\end{tabular}

Extraction Method: Principal Component Analysis.

a. 5 components extracted.

Source: Researchers' Primary Data

Unfortunately, this solution is not as easy to interpret as the rotated solution (rotated component matrix). But in a nutshell, we can say that this matrix basically explains how component loadings take place for each variable prior to rotation.

\subsection{Rotated Component Matrix}

The factor pattern matrix for orthogonal rotations reports the factor loadings for each variable on the factors after rotation. Table 7 below represents the results in rotated component matrix. This matrix basically represents how both the variables are weighted for each factor, and the correlation between the variables and the factor. Because there are correlations for which possible values range from -1 to +1 , the SPSS was asked to suppress (not to print) any of the correlations that are less than 0.3 values, allowing the output to be easier to read by removing the clutter of low correlations that, probably, are not meaningful in the present study.

Table 7 Rotated Component Matrix ${ }^{a}$

\begin{tabular}{c|c|c|c|c|c}
\hline \multirow{2}{*}{ Attribute } & \multicolumn{5}{|c}{ Component } \\
\cline { 2 - 6 } & $\mathbf{1}$ & $\mathbf{2}$ & $\mathbf{3}$ & $\mathbf{4}$ & $\mathbf{5}$ \\
\hline \hline F1 & 0.738 & & & & 0.460 \\
\hline F2 & 0.581 & & & 0.327 & \\
\hline F3 & 0.539 & & & 0.458 & \\
\hline F4 & 0.517 & & 0.350 & & 0.428 \\
\hline F5 & & & 0.592 & 0.396 & \\
\hline F6 & & 0.852 & & & \\
\hline F7 & & 0.850 & & & \\
\hline F8 & 0.411 & & 0.804 & & \\
\hline F9 & & & & 0.745 & \\
\hline F10 & 0.513 & & & 0.461 & \\
\hline F11 & & & 0.639 & & \\
\hline F12 & & & & & \\
\hline
\end{tabular}




\begin{tabular}{c|c|c|c|c|c}
\hline \multirow{2}{*}{ Attribute } & \multicolumn{5}{|c}{ Component } \\
\cline { 2 - 6 } & $\mathbf{1}$ & $\mathbf{2}$ & $\mathbf{3}$ & $\mathbf{4}$ & $\mathbf{5}$ \\
\hline \hline $\mathrm{F} 13$ & 0.390 & 0.311 & 0.443 & & \\
\hline $\mathrm{F} 14$ & & & 0.332 & & 0.612 \\
\hline $\mathrm{F} 15$ & 0.671 & & & & 0.701 \\
\hline $\mathrm{F} 16$ & & & 0.732 & & 0.831 \\
\hline
\end{tabular}

Extraction Method: Principal Component Analysis.

a. Rotation converged in 8 iterations.

Source: Researchers' Primary Data

The results in the above table show that there is only one variable load on three factors while there are five variables load on two factors. Overall, using the extraction method of principal component analysis and the Varimax Rotation method with Kaiser Normalization, the factor analysis has generated five factors. The entire rotation solution converged within 8 iterations. The component transformation (rotated component) matrix is shown in Table 8.

\subsection{Component Transformation Matrix}

This matrix describes the specific rotation applied to the factor solution. Basically, this is the matrix with which the un-rotated factor matrix is multiplied to get the rotated factor matrix. It does not require to be interpreted. Table 8 (component transformation matrix) represents the correlations among different extracted factors.

Table 8 Component Transformation Matrix

\begin{tabular}{c|c|c|c|c|c}
\hline Component & $\mathbf{1}$ & $\mathbf{2}$ & $\mathbf{3}$ & $\mathbf{4}$ & $\mathbf{5}$ \\
\hline $\mathbf{1}$ & 0.708 & -0.670 & 0.052 & -0.093 & 0.195 \\
\hline $\mathbf{2}$ & 0.108 & 0.152 & 0.941 & -0.187 & -0.214 \\
\hline $\mathbf{3}$ & 0.404 & 0.394 & 0.099 & 0.786 & 0.232 \\
\hline $\mathbf{4}$ & 0.508 & 0.610 & -0.253 & -0.551 & 0.056 \\
\hline $\mathbf{5}$ & -0.256 & 0.041 & 0.197 & -0.187 & 0.927 \\
\hline
\end{tabular}

Extraction Method: Principal Component Analysis.

Rotation Method: Varimax with Kaiser Normalization.

Source: Researchers' Primary Data

Principal component analysis was the extraction method used and Varimax with Kaiser Normalization as the rotation method used in generating the above solution matrix. 


\section{Results and Discussion}

In line to the objectives of the present study, the desirable attributes (the variables) of TSBs were formulated and explored to understand how taxpayers, as investors, perceive TSBs as an investment avenue while, through tax exemptions, reducing their total tax liabilities or part of it. There were total 16 such critical attributes related to TSBs on which the study was performed. All of these attributes weighed heavily on the taxpayer to influence their decision towards such investments. To make the outcomes of the study more meaningful, a statistical application of factor analysis, using SPSS, was performed on the primary data collected through a questionnaire-based survey. The complete set of critical attributes was then divided into five critical factors, able to represent the multiple outcomes of the variables (the attributes). These five resulting critical factors and the grouped variables are shown below:

\section{Result: Factor 1}

It includes the five attributes of TSBs "Expertise of Seller Firm", "Past Performance of Firm", "Reputation of Seller Firm", "Promptness in Updating Customer", and "Resolution of Customer Complaints". It may be inferred that these attributes are weighing heavily on the "Selling Firm" side and hence can be grouped (named) as "Selling Firm Factor".

\section{Result: Factor 2}

This factor includes the two variables i.e. "Services and Supports" and "Transparency in Information Disclosure". Since these variables can be grouped together into operations, services and sales supports, this factor can be renamed as "Services and Supports Factor"

\section{Result: Factor 3}

This factor was weighed heavily by the variables "Cost of Buying Assets", "Credit Ratings of Tax Saving Bonds", "Liquidity in Investment", and "Compulsory Lock in Period more than 3 years". Since these variables are product specific, this factor can be named as "Product Features Factor".

\section{Result: Factor 4}

This factor consists of the four variables, e.g. "Fringe Benefits", and "Early Bird Incentives". Since these variables can be clubbed together in a group of additional benefits, the factor can be renamed as "Additional Benefits Factor"

\section{Result: Factor 5}

This is the last factor which represents the four variables, "Risk in Investment", "Tax Benefits from Investment", and "Return from Investment". Since all of these 
variables are investment and return benefits specific variables, this factor can be named as "Investment Benefit Factor".

\section{Conclusion}

The present study is an attempt to explore into the factors that affect or influence the decisions of the taxpayers (from the Aligarh district in the West Uttar Pradesh State of India) towards designated tax saving bonds, offered for exemption in tax through investments. Further, an attempt is made to explore the extent to which investors (the taxpayers) are satisfied with the different benefits offered by TSBS offering banks and other institutions/firms.

It is observed that most taxpayers were balanced between positive and negative attitudes. The variables, upon factor analysis, are grouped together in such a way which results into five factors. The factors (or the variable groups) are Selling Firm Factor, Service and Supports Factor, Product Features Factor, Additional Benefits Factor, and Investment Benefit Factors. The firms and banks selling such financial instruments might find the results of this study useful in order to enhance/develop business strategies.

The results in the study reveal that attributes related to tax saving bonds such as "Tax Benefits from the Investment in TSBs", "Return from the Investment" and "Promptness in updating customers" have higher means and which is obvious because the primary concern to most investors is the benefits from the investment and after sales service quality of the firms. The attributes "Risk in Investments" and "Compulsory Lock-in Period more than 3 years" have lowest means which indicate that these attributes related to the TSBs are seen negatively by most tax payers for the reasons that, probably, the investors do not want to take any risk in their investments in bonds and longer lock-in period will mean that their money will be stuck in such an investment for a much longer period. Another attribute "Cost of Buying Assets, the TSBs" is also seen as a negative response by the respondents, which may be due to the reason that if cost of buying goes up, the yield on the return comes down.

In future, research can be conducted in other regions/states and onto a larger population of taxpayers to draw a bigger conclusion. Also, studies can be conducted to find out the taxpayers' interests in investing in various other options available within TSBs (Infra bonds, tax free bonds etc).

The Reserve Bank of India allows Foreign Institutional Investors (FIIs) to invest up to USD 25 billion in infrastructure bonds and debentures of Indian infrastructure companies. Since FIIs are participating heavily in infrastructure developments in India, how Indian investors including taxpayers see it from the perspective of investment and also being part of development of the country is a question for which an answer has to be found. Future research on this topic might explore this. 


\section{References}

Athma, P. and Kumar, R. (2007). An Explorative Study of Life Insurance Purchase Decision Making: Influence of Product and Non-Product Factors. ICFAI Journal of Risk \& Insurance, 4(3), pp. 19-21.

Cronbach, L. J. (1951). Coefficient Alpha and the Internal Structure of Tests. Psychometrika, 16(3), pp. 297-334. DOI: http://dx.doi.org/10.1007/BF02310555

Clark, G. L., Almond, S. and Strauss, K. (2012) The Home, Pension Savings and Risk Aversion: Intentions of the Defined Contribution Pension Plan Participants of a London-Based Investment Bank at the Peak of the Bubble. Urban Studies, 49(6), pp. 1251-1273. DOI: http://dx.doi.org/10.1177/0042098011410332

Dash, G. and Sood, T. (2013). Why Should One Invest in a Life Insurance Product? An Empirical Study. Researchers World, 4(1), pp. 36-45.

Dick, A. S. and Basu, K. (1994). Customer Loyalty: Toward an Integrated Conceptual Framework. Journal of the Academy of Marketing Science, 22(2), pp. 99-113.

George, D. and Mallery, P. (2012). SPSS for Windows Step by Step: A Simple Guide and Reference, 17.0 Update, $10^{\text {th }}$ Editon, Pearson Education: India.

Gerpott, T., Rams, W. and Schindler, A. (2001). Customer Retention, Loyalty, and Satisfaction in the German Mobile Cellular Telecommunications Market. Telecommunication Policy, 25(4), pp. 46-59.

Gordon, S. (1991). U. S. Savings Bonds for Investment Purposes. The CPA Journal, 61(12), pp. 76-79.

Mahesh, P. (2012, January 11). How to Choose the Best Infrastructure Bonds this Tax-Saving Season Analysis. The Economic Times (Online), Retrieved from http://search.proquest.com/docview/914961413.

Parasuraman, A. et al. (1985). A Conceptual Model of Service Quality and Its Implications for Future Research. Journal of Marketing, 49(4), pp. 41-50.

Santos, J. Reynaldo A. (1999). Cronbach's Alpha: A Tool for Assessing the Reliability of Scales, Journal of Extension, 37(2). http://www.joe.org/joe/1999 april/tt3.php - Accessed on August 16 ${ }^{\text {th }}, 2012$.

Singh, J. and Chander, S. (2004). An Empirical Analysis of Perceptions of Investors towards Mutual Funds, Finance India, 18(4), pp. 1673-1692.

Vardhini C. P. (2012, February 11). Choosing between Tax-Saving and Tax-Free Bonds. Businessline, Retrieved from http://search.proquest.com/docview/ 1322140719. 


\section{Appendix 1 (sample questions from questionnaire)}

\section{Questionnaire: Section 2}

There are 16 questions in this section and each question has 5 options to choose from:

a. Totally Disagree - If you do not agree at all with the question.

b. Disagree - If you partially disagree.

c. Neutral - If you are either neutral or not sure whether to agree or disagree.

d. Agree - If you partially agree.

e. Totally Agree - If you $100 \%$ agree.

Please answer all the questions one by one by ticking ONLY one option.

1. You are willing to take risks in the investment you make in Tax Saving Bonds:

\begin{tabular}{|l|l|l|l|l|}
\hline Totally Disagree & Disagree & Neutral & Agree & Totally Agree \\
\hline
\end{tabular}

2. You are looking for a better return from investments as compared to tax savings:

\begin{tabular}{|l|l|l|l|l|}
\hline Totally Disagree & Disagree & Neutral & Agree & Totally Agree \\
\hline
\end{tabular}

Thank You for Your Time and Patience for Completing the Survey. 